PROCEEDINGS OF THE

AMERICAN MATHEMATICAL SOCIETY

Volume 139, Number 2, February 2011, Pages 707-720

S 0002-9939(2010)10535-X

Article electronically published on August 19, 2010

\title{
DETERMINISTIC THINNING OF FINITE POISSON PROCESSES
}

\author{
OMER ANGEL, ALEXANDER E. HOLROYD, AND TERRY SOO
}

(Communicated by Edward C. Waymire)

\begin{abstract}
Let $\Pi$ and $\Gamma$ be homogeneous Poisson point processes on a fixed set of finite volume. We prove a necessary and sufficient condition on the two intensities for the existence of a coupling of $\Pi$ and $\Gamma$ such that $\Gamma$ is a deterministic function of $\Pi$, and all points of $\Gamma$ are points of $\Pi$. The condition exhibits a surprising lack of monotonicity. However, in the limit of large intensities, the coupling exists if and only if the expected number of points is at least one greater in $\Pi$ than in $\Gamma$.
\end{abstract}

\section{INTRODUCTION}

Given a homogeneous Poisson point process on $\mathbb{R}^{d}$, it is well known that selecting each point independently with some fixed probability gives a homogeneous Poisson process of lower intensity. This is often referred to as thinning. Ball [1] proved the surprising fact that in $d=1$, thinning can be achieved without additional randomization: we may choose a subset of the Poisson points as a deterministic function of the Poisson process so that the chosen points form a Poisson process of any given lower intensity; furthermore, the function can be taken to be a translationequivariant factor (that is, if a translation is applied to the original process, the chosen points are translated by the same vector). Holroyd, Lyons and Soo 7 extended this result to all dimensions $d$ and further strengthened it by showing that the function can be made isometry-equivariant and that the non-chosen points can also form a Poisson process (it cannot be independent of the process of chosen points, however). Evans [3] proved that a Poisson process cannot be similarly thinned in an equivariant way with respect to any group of affine measure-preserving maps that is strictly larger than the isometry group.

Here we address questions of deterministic thinning for a Poisson process in a finite volume. Postponing considerations of equivariance, we simply ask whether there exists a deterministic thinning rule giving a Poisson process of lower intensity. The answer depends on the two intensities, as follows. Let $\mathcal{L}$ denote Lebesgue measure on $\mathbb{R}^{d}$.

Received by the editors December 2, 2009 and, in revised form, December 4, 2009 and April 12, 2010

2010 Mathematics Subject Classification. Primary 60G55.

Funded in part by Microsoft Research (AEH) and NSERC (all authors).

(C)2010 American Mathematical Society 
Theorem 1. Fix $\lambda>\mu>0$ and a Borel set $S \subset \mathbb{R}^{d}$ with $\mathcal{L} S \in(0, \infty)$. Let $\Pi$ be a homogeneous Poisson process of intensity $\lambda$ on $S$. Let $X$ and $Y$ be Poisson random variables with respective means $\lambda \mathcal{L} S$ and $\mu \mathcal{L} S$. The following are equivalent.

(i) There exists a measurable function $f$ such that $f(\Pi)$ is a homogeneous Poisson process of intensity $\mu$ on $S$, and every point of $f(\Pi)$ is a point of $\Pi$ almost surely.

(ii) There exists an integer $k \geq 0$ such that

$$
\begin{aligned}
\mathbb{P}(X=k) & \leq \mathbb{P}(Y=k), \\
\text { and } \quad \mathbb{P}(X \leq k+1) & \leq \mathbb{P}(Y \leq k) .
\end{aligned}
$$

(iii) There is no integer $k \geq 0$ such that

$$
\begin{aligned}
& \mathbb{P}(X=k+1)>\mathbb{P}(Y=k+1), \\
\text { and } \quad & \mathbb{P}(X \leq k+1)>\mathbb{P}(Y \leq k) .
\end{aligned}
$$

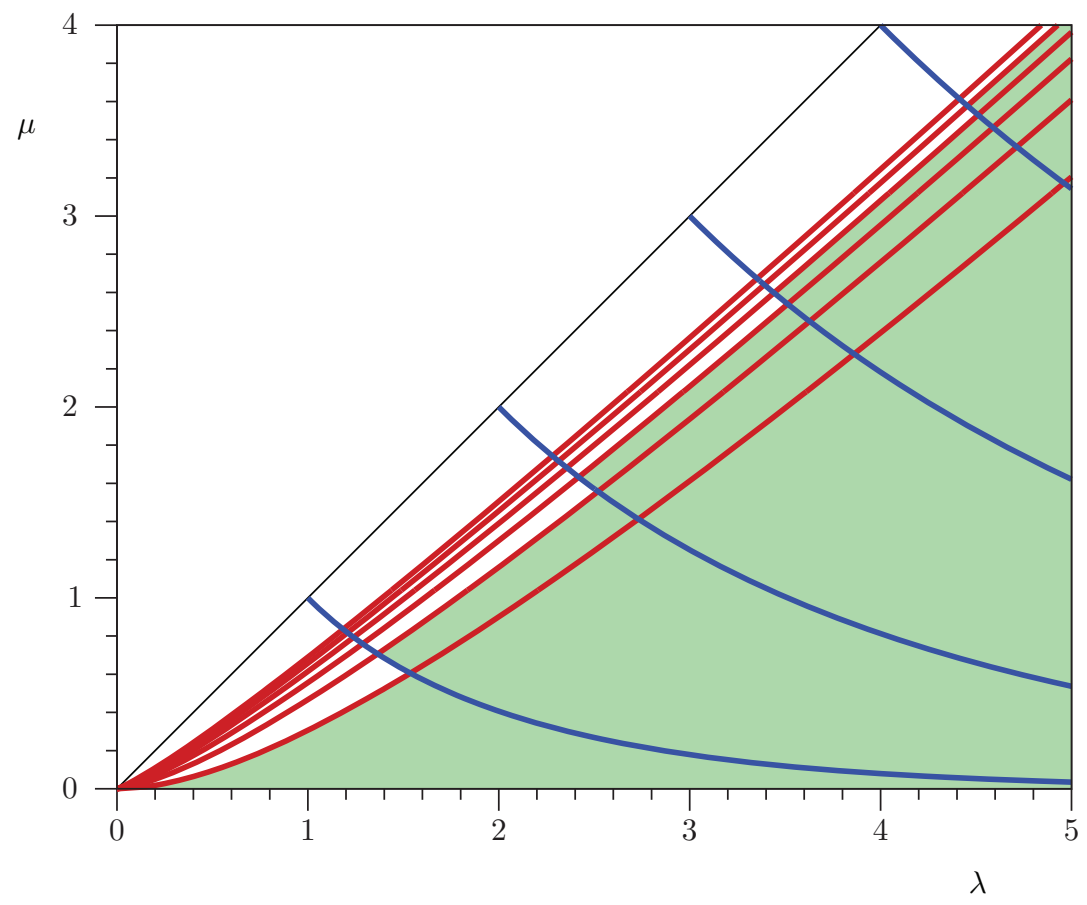

Figure 1. The shaded (closed) region is the set of pairs of intensities $(\lambda, \mu)$ for which a thinning exists in the case $\mathcal{L} S=1$. Also shown are the curves $\mathbb{P}(X \leq k+1)=\mathbb{P}(Y \leq k)$ for $k=0, \ldots, 5$ (red), the curves $\mathbb{P}(X=k)=\mathbb{P}(Y=k)$ for $k=1, \ldots, 4$ (blue), and the line $\mu=\lambda$.

Figure 1 depicts the pairs $(\lambda, \mu)$ for which conditions (i)-(iii) hold. If $f$ satisfies condition (ii) of Theorem 1, we say that $f$ is a (deterministic, Poisson) thinning on $S$ from $\lambda$ to $\mu$. The domain and range of $f$ are both the set of simple point measures on $S$. The equivalence of (ii) and (iii) is of course a relatively mundane technicality, but it is useful to have both forms of the condition available. 
Remark 1. By the Borel isomorphism theorem (see e.g. [10, 3.4.24]) and the mapping theorem [6], Theorem 1 generalizes immediately to any standard Borel space $S$ with a finite non-atomic measure $\ell$ in place of $\mathcal{L}$. By the same token, it suffices to prove Theorem 1 for the special case $S=[0,1]$.

To be precise, the above remark may be justified as follows. Observe first that by scaling $\lambda$ and $\mu$ we may assume that $\ell(S)=1$. Let $\phi: S \rightarrow[0,1]$ be a Borel isomorphism; that is, $\phi$ is a measurable bijection so that $\ell \circ \phi^{-1}(B)=\mathcal{L}(B)$ for all Borel sets $B \subset[0,1]$. Such $\phi$ (and $\phi^{-1}$ ) act naturally on point processes by $\phi(\Pi)=$ $\Pi \circ \phi$. If $\Pi$ is a homogeneous Poisson point process on $S$, then by the mapping theorem, $\phi(\Pi)$ is a homogeneous Poisson point process of the same intensity on $[0,1]$. Thus $f$ is a thinning from $\lambda$ to $\mu$ on $S$ if and only if $\phi \circ f \circ \phi^{-1}$ is a thinning from $\lambda$ to $\mu$ on $[0,1]$.

The corollaries below follow from Theorem 1 by an analysis of the curves in Figure 1.

Corollary 2 (Monotonicity in $\lambda$ ). Suppose there is a thinning from $\lambda$ to $\mu$ on $[0,1]$.

(i) If $\lambda^{\prime}>\lambda$, then there exists a thinning from $\lambda^{\prime}$ to $\mu$.

(ii) If $\mu^{\prime} / \lambda^{\prime}=\mu / \lambda$ and $\lambda^{\prime}>\lambda$, then there exists a thinning from $\lambda^{\prime}$ to $\mu^{\prime}$.

Corollary 3 (Non-monotonicity in $\mu$ ). There are positive real numbers $\lambda>\mu>\mu^{\prime}$ such that there exists a thinning from $\lambda$ to $\mu$ but not from $\lambda$ to $\mu^{\prime}$.

Corollary 3 may come as a surprise. However, it follows from Theorem 1 by a numerical computation or an inspection of Figure 1 . In particular, an example with $\mathcal{L} S=1$ is $\left(\lambda, \mu, \mu^{\prime}\right)=(1.45,0.7,0.6)$ (as may be checked by taking $k=1$ in Theorem 1 (iii) and $k=0$ in (iii)). Furthermore, there are examples satisfying $\lambda=n+1 / 2+o(1)$ and $\mu, \mu^{\prime}=n-1 / 2+o(1)$ as $n \rightarrow \infty$.

For $\mu>0$ define

$$
\lambda_{c}(\mu):=\inf \{\lambda>\mu \text { : there is a thinning from } \lambda \text { to } \mu \text { on }[0,1]\} .
$$

By Theorem 1 (iii) and Corollary 2 (ii), there exists a thinning from $\lambda$ to $\mu$ if and only if $\lambda \geq \lambda_{c}(\mu)$.

The next corollary states that there exists a thinning if the average number of points to be deleted is at least one, while the converse holds in asymptotic form.

Corollary 4 (Asymptotic threshold). We have $\lambda_{c}(\mu) \leq \mu+1$ for all $\mu>0$, and $\lambda_{c}(\mu) \geq \mu+1-o(1)$ as $\mu \rightarrow \infty$

Our proof of Theorem 1 is constructive in the sense that for $\lambda$ and $\mu$ satisfying conditions (ii) or (iii) (and for $S=[0,1]$, say), our argument gives an explicit thinning $f$ satisfying condition (i). The construction relies on the following key result, which states that given $n$ unordered uniformly random points in an interval, we may deterministically delete one of them in such a way that the remaining $n-1$ are again uniformly random. Write $B^{\{n\}}$ for the set of all subsets of $B$ of size $n$.

Proposition 5 (One-point deletion). Let $U_{1}, \ldots, U_{n}$ be independent and identically distributed (i.i.d.) random variables uniform on $[0,1]$, and define the random set $\mathcal{U}:=\left\{U_{1}, \ldots, U_{n}\right\}$. There exists a measurable function $g:[0,1]^{\{n\}} \rightarrow[0,1]^{\{n-1\}}$ such that $g(A) \subset A$ for all $A$ and

$$
g(\mathcal{U}) \stackrel{d}{=}\left\{U_{1}, \ldots, U_{n-1}\right\} .
$$

Moreover, there exists a measurable $v:[0,1]^{\{n\}} \rightarrow[0,1]$ such that $v(\mathcal{U})$ is uniform on $[0,1]$ and is independent of $g(\mathcal{U})$. 
Even in the case $n=2$, the first claim of Proposition 5 is far from obvious and makes an entertaining puzzle. Of course, the claim would be trivial if we allowed $g$ to be a function of the ordered tuple $\left(U_{1}, \ldots, U_{n}\right)$ or a function of $\mathcal{U}$ together with an independent roll of an $n$-sided die.

The function $v$ in Proposition 5 may be thought of as extracting "spare" randomness associated with the location of the deleted point $\mathcal{U} \backslash g(\mathcal{U})$. This will be useful in the proof of Theorem 1 because it will make it easy to delete a random number of further points once one point has been deleted.

Proposition [5] is somewhat reminiscent of the following fact proved in [5] (although the proofs appear unrelated). Given a homogeneous Poisson process $\Pi$ on $\mathbb{R}^{d}$, it is possible to choose a point $W$ of $\Pi$, as a deterministic function of $\Pi$, so that deleting $W$ and translating the remaining points by $-W$ yields again a homogeneous Poisson process.

Proposition 5 motivates the search for couplings of Poisson random variables $X$ and $Y$ such that either $X=Y=0$ or $X>Y$. An important observation of Ball [1, Lemma 3.1] is that the standard "quantile coupling" (i.e. $X=F_{X}^{-1}(U)$ and $Y=F_{Y}^{-1}(U)$ where $F_{X}, F_{Y}$ are the distribution functions and $U$ is uniform on $[0,1])$ has this property provided the mean of $X$ is sufficiently large as a function of the mean of $Y$. More generally, given a coupling of Poisson random variables $X, Y$ with means $\lambda, \mu$ such that $X>Y$ except on an event $A \in \sigma(X)$ on which $X=Y$, it is not difficult to show using Proposition 5 that there exists a thinning from $\lambda$ to $\mu$. Condition (iii) of Theorem 1 implies the existence of such a coupling.

Remark 2 (Infinite volumes). In the case of infinite volumes it is easier (but still nontrivial) to show that a Poisson thinning from $\lambda$ to $\mu$ always exists when $\lambda>\mu$; see [7, Example 2]. Our results yield the following alternative construction, with the additional property that whether or not a point is deleted is determined by the process within a fixed finite radius. Partition $\mathbb{R}^{d}$ into cubes of volume $1 /(\lambda-\mu)$. By Corollary 4 there exists a thinning on each cube from $\lambda$ to $\mu$; by applying each simultaneously we obtain a thinning on all of $\mathbb{R}^{d}$.

The paper is organized as follows. In Section 2 we will prove some easier parts of Theorem 1. In Section 3 we will prove Proposition 5. In Section 4 we will define the coupling of Poisson random variables that will be used to prove the existence of a thinning. In Section 5 we will finish the proof of Theorem 1 and also prove the corollaries. Finally in Section 6 we will briefly address some variant concepts, including deterministic thinnings that are equivariant with respect to a group of isometries, and deterministic splittings, where the points of the Poisson point process are partitioned into two sets, each of which forms a Poisson point process. We will also address deterministic thickening: we show that on a finite volume, it is impossible to add points, as a deterministic function of a Poisson point process, to obtain a Poisson point process of higher intensity.

\section{Proof of Theorem 1; Easy implications}

We will prove Theorem 1 by showing that for the existence of a thinning as in (ii), condition (iii) is necessary, (iii) is sufficient, and (iii) implies (iii).

Let $\mathbb{M}$ be the space of all simple point measures on $[0,1]$. For $\nu \in \mathbb{M}$, we denote the support of $\nu$ by

$$
[\nu]:=\{x \in[0,1]: \nu(\{x\})=1\} .
$$


Let $\mathbb{N}=\{0,1, \ldots\}$. For each $n \in \mathbb{N}$, let $\mathbb{M}_{n}:=\{\nu \in \mathbb{M}: \nu([0,1])=n\}$. The following characterization is useful. A point process $\Pi$ on $[0,1]$ is a Poisson point process of intensity $\lambda$ if and only if the random variable $\Pi([0,1])$ is Poisson with mean $\lambda$, and, for each $n \in \mathbb{N}$, conditional on $\Pi \in \mathbb{M}_{n}$, the set $[\Pi]$ has the distribution of $\left\{U_{1}, \ldots, U_{n}\right\}$, where $U_{1}, \ldots, U_{n}$ are i.i.d. random variables uniformly distributed on $[0,1]$. See [8, Theorem 1.2.1] or [6] for background.

Proof of Theorem [1 (ii) $\Longrightarrow$ (iii). Let $\Pi$ be a Poisson point process on $[0,1]$ with intensity $\lambda$ and let $f$ be a thinning from $\lambda$ to $\mu$. Set $X:=\Pi([0,1])$ and $Y:=$ $f(\Pi)([0,1])$ and let $k \in \mathbb{N}$ be such that

$$
\mathbb{P}(X=k+1)>\mathbb{P}(Y=k+1) .
$$

We will show that

$$
\mathbb{P}(X=Y=k+1)=0 .
$$

In other words, if on the event $X=k+1$ the thinning $f$ sometimes deletes points of $\Pi$, then it must (almost) always delete points. Since $X \geq Y$, if $X \leq k+1$, then either $X \leq k$, in which case $Y \leq k$, or $X=k+1$, in which case (2) implies that $Y \leq k$. Thus, $\mathbb{P}(X \leq k+1) \leq \mathbb{P}(Y \leq k)$ and condition (iiii) holds.

It remains to show (2). Let $Q$ be the law of $\left\{U_{1}, \ldots, U_{k+1}\right\}$ where the $U_{i}$ are i.i.d. uniform in $[0,1]$. Let $\mathcal{J}:=\left\{\nu \in \mathbb{M}_{k+1}: f(\nu)=\nu\right\}$. Thus, $\mathcal{J}$ is a set of measures where $f$ does not delete any points. Let $[\mathcal{J}]:=\{[\nu]: \nu \in \mathcal{J}\}$, so that

$$
\mathbb{P}(\Pi \in \mathcal{J})=\mathbb{P}(X=k+1) \cdot Q([\mathcal{J}])
$$

and also

$$
\mathbb{P}(f(\Pi) \in \mathcal{J})=\mathbb{P}(Y=k+1) \cdot Q([\mathcal{J}]) .
$$

Since $\{\Pi \in \mathcal{J}\} \subseteq\{f(\Pi) \in \mathcal{J}\}$, we deduce

$$
\mathbb{P}(X=k+1) \cdot Q([\mathcal{J}]) \leq \mathbb{P}(Y=k+1) \cdot Q([\mathcal{J}]) .
$$

We see that (10) and (3) force $Q([\mathcal{J}])=0$. Hence $\mathbb{P}(\Pi \in \mathcal{J})=0$, which implies (2).

Proof of Theorem 11: (iii) $\Longrightarrow$ (iii). Since $\lambda>\mu$ we have $\mathbb{P}(X=0)<\mathbb{P}(Y=0)$, and since $\sum_{i \in \mathbb{N}} \mathbb{P}(X=i)=\sum_{i \in \mathbb{N}} \mathbb{P}(Y=i)=1$, there exists a minimal integer $k \geq 0$ such that

$$
\mathbb{P}(X=k+1)>\mathbb{P}(Y=k+1) .
$$

By condition (iii) we must have that

$$
\mathbb{P}(X \leq k+1) \leq \mathbb{P}(Y \leq k) .
$$

By the minimality of $k$ we have that

$$
\mathbb{P}(X=k) \leq \mathbb{P}(Y=k) .
$$

It remains to prove that (iii) implies (ii) in Theorem 1, which we will do in Section 5 after assembling the necessary tools. Our strategy for constructing the thinning $f$ will be as follows. If the number of points $\Pi(S)$ is at most $k$, we retain all of them; otherwise, we first delete one point using Proposition 5, then delete a suitable random number of others. 


\section{DELETING UNIFORM RANDOM VARIABLES}

We will give two proofs of Proposition 5. Our original proof is given in Section 6 and gives a function $g$ with an additional rotation-equivariance property. The proof below follows a suggestion of Michael Brand; a version appears on his web page of mathematical puzzles [2, March 2009]. Both proofs rely on the following observation.

Lemma 6. Let $Q$ be a probability measure on an arbitrary Borel space $S$. Let $Q_{m}$ be the law of $\left\{U_{1}, \ldots, U_{m}\right\}$, where the $U_{i}$ are i.i.d. with law $Q$. Let $g: S^{\{n\}} \rightarrow S^{\{n-1\}}$ be a measurable function such that $g(A) \subset A$ for all $A$, and define for $B \in S^{\{n-1\}}$,

$$
R(B)=\{w \in S: g(B \cup\{w\})=B\} .
$$

If for $Q_{n-1}$-a.e. $B \in S^{\{n-1\}}$ we have $Q(R(B))=n^{-1}$, then $Q_{n} \circ g^{-1}=Q_{n-1}$.

Proof. We prove the following stronger fact: without any assumption on $Q(R(B))$, the Radon-Nykodim derivative satisfies

$$
\frac{d\left(Q_{n} \circ g^{-1}\right)}{d Q_{n-1}}=n Q(R(\cdot)), \quad Q_{n-1} \text {-a.e. }
$$

Let $U_{1}, \ldots, U_{n}$ be i.i.d. with law $Q$ and write $\mathcal{U}_{m}=\left\{U_{1}, \ldots, U_{m}\right\}$. Let $\mathcal{A} \subseteq$ $S^{\{n-1\}}$ be measurable. Since the $U_{i}$ are exchangeable,

$$
Q_{n} \circ g^{-1}(\mathcal{A})=\mathbb{P}\left(g\left(\mathcal{U}_{n}\right) \in \mathcal{A}\right)=n \mathbb{P}\left(g\left(\mathcal{U}_{n}\right)=\mathcal{U}_{n-1} \in \mathcal{A}\right) .
$$

We have the identity of events

$$
\left\{g\left(\mathcal{U}_{n}\right)=\mathcal{U}_{n-1} \in \mathcal{A}\right\}=\left\{U_{n} \in R\left(\mathcal{U}_{n-1}\right)\right\} \cap\left\{\mathcal{U}_{n-1} \in \mathcal{A}\right\} .
$$

Therefore, since $\mathcal{U}_{n-1}, U_{n}$ have respective laws $Q_{n-1}, Q$,

$$
Q_{n} \circ g^{-1}(\mathcal{A})=\int_{\mathcal{A}} n Q(R(B)) d Q_{n-1}(B) .
$$

Proof of Proposition [5. Let $S=\{1, \ldots, n\} \times[0,1] \times[0,1]$. By the Borel isomorphism theorem, there exists a measurable bijection $\phi:[0,1] \rightarrow S$ such that if $U$ is uniformly distributed in $[0,1]$, then $\phi(U)$ is uniformly distributed in $S$. Thus we may assume that the $U_{i}$ are i.i.d. uniform in $S=\{1, \ldots, n\} \times[0,1] \times[0,1]$ instead of in $[0,1]$, and write $U_{i}=\left(X_{i}, Y_{i}, Z_{i}\right)$. Let $Q_{m}$ be as in Lemma 6 .

Let $K$ be the $\{1, \ldots, n\}$-valued random variable given by

$$
K \equiv \sum_{i=1}^{n} X_{i} \quad \bmod n
$$

Let $W=\left(X^{\prime}, Y^{\prime}, Z^{\prime}\right)$ be the element of $\mathcal{U}$ that has the $K$ th smallest $Y_{i}$. Define

$$
g(\mathcal{U})=\mathcal{U} \backslash\{W\} ; \quad v(\mathcal{U})=Z^{\prime}
$$

Since the $X_{i}$ 's, $Y_{i}$ 's and $Z_{i}$ 's are all independent it is clear that $v(\mathcal{U})$ is uniform on $[0,1]$ and independent of $g(\mathcal{U})$. It remains to show that $g(\mathcal{U})$ has law $Q_{n-1}$.

To see this, let $B=\left\{\left(x_{1}, y_{1}, z_{1}\right), \ldots,\left(x_{n-1}, y_{n-1}, z_{n-1}\right)\right\}$, and let $k$ be such that $y^{\prime} \in[0,1]$ is the $k$ th smallest element of $\left\{y_{1}, \ldots, y_{n-1}, y^{\prime}\right\}$. There is a unique $x^{\prime} \in\{1, \ldots, n\}$ so that $k \equiv x^{\prime}+x_{1}+\cdots+x_{n-1}(\bmod n)$. It follows from the definition of $K$ and $W$ that if $B \in S^{n-1}$, then for a.e. $y^{\prime}, z^{\prime} \in[0,1]$ there is a unique $w=\left(x^{\prime}, y^{\prime}, z^{\prime}\right) \in S$ so that $g(B \cup\{w\})=B$. Thus

$$
Q_{1}\{w \in S: g(B \cup\{w\})=B\}=n^{-1},
$$


and by Lemma 6. $Q_{n}\left(g^{-1}(\cdot)\right)=Q_{n-1}(\cdot)$, as required.

Remark 3. For a constructive proof of Proposition [5, in place of the bijection $\phi$ we may use the map (a bijection up to null sets) $x \mapsto\left(1+\lfloor n x\rfloor,\{n x\}_{\mathcal{E}},\{n x\}_{\mathcal{O}}\right.$ ), where for $x \in \mathbb{R}$ we write $\lfloor x\rfloor,\{x\}$ for the integer part and fractional part respectively, and $x_{\mathcal{E}}, x_{\mathcal{O}}$ for the numbers with binary expansions obtained by taking respectively the even- and odd-indexed digits from the expansion of $x$.

The following corollary of Proposition 5 states how the "spare" randomness will be utilized in the proof of Theorem 1. Write $B^{\{<n\}}$ for the set of all subsets of $B$ of size strictly less than $n$.

Corollary 7. Let $U_{1}, \ldots, U_{n}$ be i.i.d. random variables uniformly distributed on $[0,1]$, and define the random set $\mathcal{U}:=\left\{U_{1}, \ldots, U_{n}\right\}$. Let $Z$ be any $\{0, \ldots, n-1\}$ valued random variable that is independent of $\mathcal{U}$. There exists a measurable function $h:[0,1]^{\{n\}} \rightarrow[0,1]^{\{<n\}}$ such that $h(A) \subset A$ for all $A$ and

$$
h(\mathcal{U}) \stackrel{d}{=}\left\{U_{1}, \ldots, U_{Z}\right\} .
$$

Proof. Define the random set $\mathcal{U}_{n-1}:=\left\{U_{1}, \ldots, U_{n-1}\right\}$. Let $V$ be uniformly distributed on $[0,1]$ and independent of $\left(U_{1}, \ldots, U_{n-1}\right)$. Since $Z<n$, there exists a measurable $\widehat{h}:[0,1]^{\{n-1\}} \times[0,1] \rightarrow[0,1]^{\{<n\}}$ such that $\widehat{h}\left(\mathcal{U}_{n-1}, V\right) \stackrel{d}{=}\left\{U_{1}, \ldots, U_{Z}\right\}$ and $\widehat{h}\left(\mathcal{U}_{n-1}, V\right) \subseteq \mathcal{U}_{n-1}$; to construct such an $\widehat{h}$, use $V$ to randomly order $\mathcal{U}_{n-1}$ and independently construct $Z$ with the correct distribution, and then select the first $Z$ points in the ordering. Now let $g$ and $v$ be as in Proposition 5, so that $(g(\mathcal{U}), v(\mathcal{U})) \stackrel{d}{=}\left(\mathcal{U}_{n-1}, V\right)$. Define $h(\mathcal{U}):=\widehat{h}(g(\mathcal{U}), v(\mathcal{U}))$.

\section{Couplings of Poisson random variables}

In this section we will show that condition (iii) of Theorem 1 implies the existence of a certain coupling of Poisson random variables that will be used to construct thinnings.

We need the following simple result which implies that each of the two families of curves in Figure 1 is non-intersecting.

Lemma 8 (Non-intersection). Let $X, Y$ be Poisson random variables with respective means $\lambda, \mu$, where $\lambda>\mu$. For every integer $k \geq 0$,

(i) $\mathbb{P}(X=k+1) \leq \mathbb{P}(Y=k+1)$ implies $\mathbb{P}(X=k) \leq \mathbb{P}(Y=k)$;

(ii) $\mathbb{P}(X \leq k+1) \leq \mathbb{P}(Y \leq k)$ implies $\mathbb{P}(X \leq k+2) \leq \mathbb{P}(Y \leq k+1)$.

The following fact will be useful in the proof of Lemma 8 , and elsewhere. If $X$ is a Poisson random variable with mean $\lambda$, then $\mathbb{P}(X \leq n)$ is the probability that the $(n+1)$ st arrival in a standard Poisson process occurs after time $\lambda$, so

$$
\mathbb{P}(X \leq n)=\frac{1}{n !} \int_{\lambda}^{\infty} e^{-t} t^{n} d t .
$$

Proof of Lemma 8. Let $X, Y$ be Poisson with respective means $\lambda, \mu$, where $\lambda>\mu$. Part (i) is easy to check:

$$
\begin{aligned}
\mathbb{P}(X=k) & =\frac{k+1}{\lambda} \mathbb{P}(X=k+1) \\
& \leq \frac{k+1}{\lambda} \mathbb{P}(Y=k+1)=\frac{\mu}{\lambda} \mathbb{P}(Y=k)<\mathbb{P}(Y=k) .
\end{aligned}
$$


For (iii), using (4), the following inequalities are all equivalent:

$$
\begin{aligned}
\mathbb{P}(X \leq k+1) & \leq \mathbb{P}(Y \leq k) \\
\mathbb{P}(X \leq k+1) & \leq \mathbb{P}(Y \leq k+1)-\mathbb{P}(Y=k+1) \\
\frac{1}{(k+1) !} \int_{\lambda}^{\infty} e^{-t} t^{k+1} d t & \leq \frac{1}{(k+1) !} \int_{\mu}^{\infty} e^{-t} t^{k+1} d t-\frac{1}{(k+1) !} e^{-\mu} \mu^{k+1} ; \\
e^{-\mu} \mu^{k+1} & \leq \int_{\mu}^{\lambda} e^{-t} t^{k+1} d t ; \\
1 & \leq \int_{\mu}^{\lambda} e^{\mu-t}\left(\frac{t}{\mu}\right)^{k+1} d t
\end{aligned}
$$

But the right side of the last inequality is clearly increasing in $k$.

Corollary 9 (Monotone coupling). If condition (iii) of Theorem 1 is satisfied by Poisson random variables $X$ and $Y$ and an integer $k$, then there exists a coupling of $X$ and $Y$ with the following properties:

(i) The coupling is monotone; that is $X \geq Y$.

(ii) If $X \leq k$, then $X=Y$.

(iii) If $X>k$, then $X>Y$.

Before proving Corollary 9 we recall that if $W$ and $V$ are real-valued random variables, we say that $W$ stochastically dominates $V$ if $\mathbb{P}(W \leq x) \leq \mathbb{P}(V \leq x)$ for all $x \in \mathbb{R}$. If $W$ stochastically dominates $V$, then there exists a random variable $V^{\prime}$ (on a possibly larger probability space) such that $W \geq V^{\prime}$ and $V^{\prime}$ has the same law as $V$; more specifically, such a $V^{\prime}$ may be defined so that $W$ and $V^{\prime}$ have the same joint law as the quantile coupling of $W$ and $V$ as mentioned in the introduction. See e.g. [12, Chapter 1] and [11] for background.

Proof of Corollary 9, Let $X$ and $Y$ be Poisson random variables that satisfy condition (iii) of Theorem 1 with some integer $k$. Applying Lemma 8 , we obtain that

$$
\mathbb{P}(X=j) \leq \mathbb{P}(Y=j) \text { for all } 0 \leq j \leq k
$$

and

$$
\mathbb{P}(X \leq j+1) \leq \mathbb{P}(Y \leq j) \text { for all } j \geq k .
$$

By (5), we may define a probability mass function $m$ on $\mathbb{N}$ as follows:

$$
m(j):= \begin{cases}\mathbb{P}(Y=0)-\mathbb{P}(X=0)+\mathbb{P}(X \leq k) & j=0 ; \\ \mathbb{P}(Y=j)-\mathbb{P}(X=j) & 1 \leq j \leq k ; \\ \mathbb{P}(Y=j) & j>k .\end{cases}
$$

Let $V$ be a random variable with mass function $m$. Also let $W:=(X-1) \mathbf{1}_{X>k}$. By (5) and (6), it is straightforward to check that $W$ stochastically dominates $V$, so we may assume that $W \geq V$. On $X \leq k$ we have $W=0$ and therefore $V=0$; hence we have the equality $V=V \mathbf{1}_{X>k}$. Now define a random variable

$$
Y^{\prime}:=X \mathbf{1}_{X \leq k}+V \mathbf{1}_{X>k} \text {. }
$$

The mass function of $Y^{\prime}$ is obtained by adding those of $X \mathbf{1}_{X \leq k}$ and $V$, except at 0 , and it follows that $Y^{\prime} \stackrel{d}{=} Y$. Therefore we may assume that $Y^{\prime}=Y$. On the other hand we may write

$$
X=X \mathbf{1}_{X \leq k}+(W+1) \mathbf{1}_{X>k} .
$$


By comparing the last two displays it is evident that the required properties (ii) and (iii) hold, and (i) is a consequence of them.

\section{The thinning And PRoOfs of COROLlaries}

Proof of Theorem 1. (iii) $\Longrightarrow$ (ii). Assuming condition (iii) we construct a thinning $f$. Let $k$ be an integer satisfying condition (iii). Let $\Pi$ be a Poisson point process on $[0,1]$ with intensity $\lambda$. Write $X=\Pi([0,1])$; thus $X$ is a Poisson random variable with mean $\lambda$. Let $Y$ be a coupled Poisson random variable with mean $\mu$ so that $X$ and $Y$ satisfy the conclusion of Corollary 9 . We will define $f$ so that $f(\Pi)([0,1]) \stackrel{d}{=} Y$.

For each $n \geq 0$, let $Q_{n}$ be the law of $Y$ conditional on $X=n$. Let $Z_{n}$ be independent of $\Pi$ and have law $Q_{n}$. By Corollary 9, if $n>k$, then $Y<n$ a.s. For each $n>k$, let $h_{n}:[0,1]^{\{n\}} \rightarrow[0,1]^{\{<n\}}$ be the function from Corollary 7 corresponding to the random variable $Z_{n}$. Let $f$ be defined by:

$$
[f(\Pi)]:= \begin{cases}{[\Pi]} & \text { if } X \leq k ; \\ h_{n}([\Pi]) & \text { if } X=n>k .\end{cases}
$$

By Corollary 9, we have $f(\Pi)([0,1]) \stackrel{d}{=} Y$. In addition, from Corollary 7$]$ we have that for all $m \geq 0$, conditional on the event that $f(\Pi)([0,1])=m$, the $m$ points of $f(\Pi)$ have the distribution of $m$ unordered i.i.d. random variables uniformly distributed on $[0,1]$ (this holds even if we condition also on $\Pi([0,1])$ ). Thus $f(\Pi)$ is a Poisson point process of intensity $\mu$ on $[0,1]$.

Proof of Corollary 2, Let $F_{\lambda}$ be the distribution function of a Poisson random variable with mean $\lambda$. Part (ii) follows immediately from Theorem 1 condition (iii) and the facts that $F_{\lambda}(k)$ is decreasing in $\lambda$ for all $k \geq 0$ and that $e^{-\lambda} \lambda^{k} / k$ ! is unimodal as a function of $\lambda$.

Let $(\lambda, \mu)$ and $\left(\lambda^{\prime}, \mu^{\prime}\right)$ satisfy the conditions of Corollary 2 part (iii). By Theorem 1 condition (iii), it suffices to show that if for some fixed $k \geq 0$, the pair $(\lambda, \mu)$ satisfies $F_{\lambda}(k+1) \leq F_{\mu}(k)$ and $e^{-\lambda} \lambda^{k} \leq e^{-\mu} \mu^{k}$, then the pair $\left(\lambda^{\prime}, \mu^{\prime}\right)$ satisfies the same inequalities (with the same $k$ ). Let $p:=\mu / \lambda$. By a variant of the argument in the proof of Lemma 8 (iii), we have that $F_{\lambda}(k+1) \leq F_{\mu}(k)$ if and only if

$$
e^{-\lambda} \lambda^{k+1} \leq(k+1) \int_{\mu}^{\lambda} e^{-t} t^{k} d t
$$

By the change of variables, $t=\lambda s$, we see that (7) is equivalent to

$$
1 \leq(k+1) \int_{p}^{1} e^{(1-s) \lambda} s^{k} d s
$$

The right side of (8) is increasing in $\lambda$. Since $\mu^{\prime} / \lambda^{\prime}=p$ and $\lambda^{\prime}>\lambda$, we have $F_{\lambda^{\prime}}(k+1) \leq F_{\mu^{\prime}}(k)$. Simple calculations show that $e^{-\lambda} \lambda^{k} \leq e^{-\mu} \mu^{k}$ if and only if

$$
\lambda \geq \frac{-k \log p}{1-p} .
$$

The left side of (9) is obviously increasing in $\lambda$. Thus we have that $e^{-\lambda^{\prime}}\left(\lambda^{\prime}\right)^{k} \leq$ $e^{-\mu^{\prime}}\left(\mu^{\prime}\right)^{k}$. 
Proof of Corollary 4. First we show that $\lambda_{c}(\mu) \leq \mu+1$. By Corollary [2 (i), it suffices to show that if $\lambda=\mu+1$, then there is a thinning from $\lambda$ to $\mu$. By Theorem 1 condition (iii), we must show for some $k \in \mathbb{N}$ that $F_{\lambda}(k+1) \leq F_{\mu}(k)$ and $e^{-\lambda} \lambda^{k} \leq e^{-\mu} \mu^{k}$. The latter condition is satisfied by choosing $k=\lfloor 1 / \log (1+1 / \mu)\rfloor$. As in the proof of Lemma 8 (ii), $F_{\lambda}(k+1) \leq F_{\mu}(k)$ if and only if

$$
\int_{\mu}^{\lambda} e^{\mu-t}\left(\frac{t}{\mu}\right)^{k+1} d t \geq 1
$$

So by the change of variables $t=\mu+s$ and the equality $\lambda=\mu+1$, it suffices to verify that

$$
\int_{0}^{1} e^{-s}\left(1+\frac{s}{\mu}\right)^{k+1} d s \geq 1
$$

Inequality (11) is a consequence of the observation that

$$
\frac{\log (1+s / \mu)}{\log (1+1 / \mu)} \geq s \text { for all } s \in[0,1]
$$

which in turn follows from $\log (\mu+s) \geq(1-s) \log \mu+s \log (\mu+1)$, an instance of the concavity of log.

Next we show that $\lambda_{c}(\mu) \geq \mu+1-o(1)$. Fix $\delta<1$, and let $\lambda=\mu+\delta$. By Theorem 1 condition (iii) it suffices to show that when $\mu$ is sufficiently large there is an integer $k$ so that $F_{\lambda}(k+1)>F_{\mu}(k)$ and $e^{-\lambda} \lambda^{k+1}>e^{-\mu} \mu^{k+1}$. The latter condition is equivalent to the inequality

$$
\left(1+\frac{\delta}{\mu}\right)^{k+1}>e^{\delta}
$$

while the former condition is equivalent to the negation of (10); moreover, by the change of variable $t=\mu+s$ this is equivalent to

$$
\int_{0}^{\delta} e^{-s}\left(1+\frac{s}{\mu}\right)^{k+1} d s<1
$$

Set $k=\lfloor\mu+1\rfloor$. For $\mu$ sufficiently large, (12) is satisfied with this $k$. Moreover, since $k+1<\mu+2$ and $(1+s / \mu)<e^{s / \mu}$, we see that the left side of (13) is bounded above by $\int_{0}^{\delta} e^{2 s / \mu} d s$, which is strictly less than 1 for $\mu$ sufficiently large.

\section{VARIANTS AND OPEN PROBLEMS}

6.1. Thickening. Theorem 1 and its corollaries address deterministic thinning, but what about deterministic thickening? Does there exist a measurable function $f$ such that if $\Pi$ is a Poisson point process on a Borel set $S$, then $f(\Pi) \geq \Pi$ and $f(\Pi)$ is a Poisson point process on $S$ of intensity higher than that of the original process $\Pi$ ? If $S$ has finite volume, then the answer is no.

Proposition 10. Fix $\lambda>0$ and Borel set $S \subset \mathbb{R}^{d}$ with $\mathcal{L}(S) \in(0, \infty)$. Let $\Pi$ be a homogeneous Poisson process of intensity $\lambda$ on $S$. There does not exist a measurable function $f$ such that $f(\Pi)$ is a homogeneous Poisson process of intensity strictly larger than $\lambda$ on $S$.

Remark 4. In Proposition 10 we do not even require that $f(\Pi) \geq \Pi$. 
Proof of Proposition 10. Let $f$ be a measurable function. Let 0 denote the zero measure. If $f(0)=0$, then $\mathbb{P}(f(\Pi)=0) \geq \mathbb{P}(\Pi=0)$ so that $f(\Pi)(S)$ cannot be a Poisson random variable of larger mean than $\Pi(S)$. If $f(0) \neq 0$, then $\mathbb{P}(f(\Pi)=f(0)) \geq \mathbb{P}(\Pi=0)>0$ so that $f(\Pi)$ gives positive mass to a single point measure other than 0 and hence cannot be a Poisson process.

By the Borel isomorphism theorem, for any Borel set $S$ of infinite volume and any $\lambda^{\prime}>0$, there exists a measurable function $f$ such that if $\Pi$ is a Poisson process of positive intensity on $S$, then $f(\Pi)$ is a Poisson point process of intensity $\lambda^{\prime}$ on $S$; but of course this does not guarantee $f(\Pi) \geq \Pi$. It is shown in [7, Theorem 3] that even in the case of infinite volume, deterministic thickening is impossible if we impose an additional finitariness condition on $f$. Gurel-Gurevich and Peled 4 have recently proved that deterministic thickening is possible if this condition is dropped.

6.2. Equivariant thinning. As remarked earlier, Theorem 1 extends immediately to any Borel space with a finite non-atomic measure. When the space has non-trivial symmetries, new questions arise.

Consider the length measure on the circle $S^{1}=\left\{x \in \mathbb{R}^{2}:\|x\|=1\right\}$. Since this measure space is isomorphic to the interval $[0,2 \pi]$ with Lebesgue measure, Theorem 1 tells us for which pairs $\lambda, \mu$ there exists a thinning. However the circle is more interesting because we can associate groups of symmetries. Given an isometry $\theta$ of $S^{1}$ and $\nu \in \mathbb{M}\left(S^{1}\right)$, let $\theta(\nu)$ be the measure given by $\theta(\nu)(A):=\nu\left(\theta^{-1}(A)\right)$ for measurable $A \subseteq S^{1}$. We say that a measurable mapping $f: \mathbb{M}\left(S^{1}\right) \rightarrow \mathbb{M}\left(S^{1}\right)$ is rotation-equivariant if $\theta(f(\nu))=f(\theta(\nu))$ for all $\nu \in \mathbb{M}\left(S^{1}\right)$ and all rotations $\theta$ of $S^{1}$. Isometry-equivariance is defined analogously.

Theorem 11. If $S$ is the unit circle $S^{1}$, and Lebesgue measure is replaced with uniform measure on $S^{1}$, then Theorem 1 holds even with the additional requirement that the thinning $f$ in condition (i) be rotation-equivariant.

Proof. The proof of Theorem 1 goes through except that we need the following rotation-equivariant version of Proposition [5] Assuming condition (iii), this allows the thinning we construct to be rotation-equivariant. We omit the rest of the details.

Proposition 12 (Equivariant deletion). Let $U_{1}, \ldots, U_{n}$ be i.i.d. random variables uniformly distributed on $S^{1}$, and define the random set $\mathcal{U}:=\left\{U_{1}, \ldots, U_{n}\right\}$. There exists a measurable function $g:\left(S^{1}\right)^{\{n\}} \rightarrow\left(S^{1}\right)^{\{n-1\}} \cup\{\emptyset\}$, with the following properties: $g$ is rotation-equivariant, $g(A) \subset A$ for any set $A$, and $g(\mathcal{U}) \stackrel{d}{=}$ $\left\{U_{1}, \ldots, U_{n-1}\right\}$. In addition, there exists a function $v:\left(S^{1}\right)^{\{n\}} \rightarrow[0,1]$ such that $v$ is rotation-invariant and $v(\mathcal{U})$ is uniformly distributed on $[0,1]$ and independent of $g(\mathcal{U})$.

Remark 5. Note that the pre-image of $\emptyset$ under the function $g$ of Proposition 12 has measure 0 . The inclusion of $\emptyset$ in the range is a technical convenience which allows $g$ to be rotation-equivariant everywhere (as supposed to almost everywhere). For example, if $A$ consists of $n$ equally spaced points in $S^{1}$, it is impossible to choose one in a rotation-equivariant way. 
To construct this function we rely on a classical problem involving fuel shortage; see e.g. [13, Gasoline Crisis]. See also Spitzer's Lemma 9, Theorem 2.1]. We repeat the problem and its solution below.

Lemma 13. Suppose a circular road has several gas stations along its length with just enough gas in total to drive a full circle around the road. Then it is possible to start at one of the stations with an empty tank and complete a circuit without running out of gas before the end.

Proof. Pretend at first we are allowed to have a negative amount of gas and still drive. Start at any point and consider the amount of gas in the car as a function of the location. After a full circle the tank is exactly empty again. Any point at which the function takes its minimum is a suitable starting point.

Proof of Proposition 12, Place $n$ gas stations at the points of $\mathcal{U} \subset S^{1}$ with gas for $1 / n$ of the circle at each. Let $z(\mathcal{U})$ be the station from which it is possible to drive around $S^{1}$ (in a counterclockwise direction); if there is more than one such station, set $z(\mathcal{U})=\emptyset$ (this has probability 0 for i.i.d. uniform points). Clearly $g(\mathcal{U}):=\mathcal{U} \backslash\{z(\mathcal{U})\}$ is rotation-equivariant.

To see that $g(\mathcal{U})$ has the claimed distribution, consider a set $B \in\left(S^{1}\right)^{\{n-1\}}$, and let $F(B) \subset S^{1}$ be the set of $x \in S^{1}$ so that $z(B \cup\{x\})=x$. By Lemma 6 , it suffices to show that $F(B)$ has measure $1 / n$ for a.e. $B$.

To see that $F(B)$ has measure $1 / n$, consider as above the amount of gas in the car (allowing a deficit) when $1 / n$ gas is placed at each point of $B$, but now continue driving indefinitely around the circle. The gas function $h(t)$ is skewperiodic: $h(t+1)=h(t)-1 / n$. Furthermore, it has derivative -1 except at points $t(\bmod 1) \in B$ where $h$ is discontinuous. It follows that there is a set $T$ of measure $1 / n$ so that $h$ attains a new minimum value at every $t(\bmod 1) \in T$. The set $T$ is exactly the set of locations where it is possible to drive a full circle starting with $1 / n$ gas, hence these are the $x$ where $z(B \cup\{x\})=x$. Note that $T$ is a finite union of intervals in $S^{1}$.

We define $v$ as follows. If $z(\mathcal{U})=\emptyset$, then set (arbitrarily) $v(\mathcal{U})=0$; otherwise, compute the set $T$ corresponding to $g(\mathcal{U})$. Given $g(\mathcal{U}), z(\mathcal{U})$ is uniformly distributed on $T$. Take the component (interval) of $T$ containing $z(\mathcal{U})$, rescale it to the interval $[0,1]$, and let $v(\mathcal{U})$ be the image of $z(\mathcal{U})$ under this rescaling.

Proposition 12 gives a deletion procedure that is equivariant to rotations, but not to other isometries of the circle (namely, reflections).

Question 1. Give necessary and sufficient conditions on $\lambda$ and $\mu$ for the existence of an isometry-equivariant thinning on the circle $S^{1}$ from $\lambda$ to $\mu$.

Remark 6. It is easy to see that in the case $n=2$, Proposition 12 would no longer hold with the group of rotations replaced by the (larger) group of isometries. Therefore, if there exists an isometry-equivariant thinning on $S^{1}$, then whenever there are exactly two points, it must either keep both of them or delete both of them. This is not always possible; for example, consider the case where $S^{1}$ is endowed with the uniform probability measure, $\lambda=2$, and $\mu=1$. If $X$ and $Y$ are Poisson random variables with means 2 and 1 respectively, then

$$
\mathbb{P}(X=2)>\mathbb{P}(Y=2) \quad \text { and } \quad \mathbb{P}(X \in\{0,2\})>\mathbb{P}(Y=0) .
$$


Hence if $f$ is a thinning on $S^{1}$ from 2 to 1 , then $f$ cannot be isometry-equivariant because whenever there are exactly two points on $S^{1}$, the first inequality implies that $f$ cannot always keep both of them, and the second inequality implies that $f$ cannot always delete both of them. However, by Theorem 11 and Corollary 4 . there exists a rotation-equivariant thinning on $S^{1}$ from 2 to 1 .

Thus the set of $(\lambda, \mu)$ for which there is an isometry-equivariant thinning on $S^{1}$ from $\lambda$ to $\mu$ is strictly smaller than the set for which there is a rotation-equivariant thinning. We do not know whether Proposition 12, with the group rotations replaced by the group of isometries, holds in the case $n \geq 4$. Ori Gurel-Gurevich has found a construction in the case $n=3$ (personal communication).

Theorem 11 can be easily generalized to some other symmetric measure spaces by using only Proposition 12, For example: the 2-sphere $S^{2}=\left\{x \in \mathbb{R}^{3}:\|x\|=1\right\}$ with the group of rotations that fix a given diameter or the torus $\mathbb{R}^{2} / \mathbb{Z}^{2}$ with translations. However, we do not know whether there exists a rotation-equivariant thinning on the sphere or an isometry-equivariant thinning on the torus.

Question 2. Give necessary and sufficient conditions on $\lambda$ and $\mu$ for the existence of a rotation-equivariant (or isometry-equivariant) thinning from $\lambda$ to $\mu$ on the 2-sphere $S^{2}$.

Similar questions about thinning can be asked in a more general setting. Let $G$ be a group of measure-preserving bijections on a standard Borel space $\mathcal{S}$ and let $\mathbb{M}(\mathcal{S})$ be the space of simple point measures on $\mathcal{S}$. We say that $f: \mathbb{M}(\mathcal{S}) \rightarrow \mathbb{M}(\mathcal{S})$ is $G$-equivariant if $f(\gamma \nu)=\gamma f(\nu)$ for all $\nu \in \mathbb{M}(\mathcal{S})$ and all $\gamma \in G$.

For the unit ball it is not difficult to show that an isometry-equivariant version of Proposition 5 holds. Indeed, since isometries of the ball preserve the norm, any selection scheme that depends only on the norms of the points will automatically be isometry-equivariant. The function $x \mapsto\|x\|^{d}$ maps a uniformly distributed random variable on the unit ball to a uniformly distributed random variable on $[0,1]$, and any thinning procedure on $[0,1]$ can be composed on this mapping. Thus for the unit ball, Theorem 1 holds even with the additional requirement that the thinning $f$ in condition (ii) be isometry-equivariant.

Question 3. For which spaces $(\mathcal{S}, G)$ is the existence of a thinning from $\lambda$ to $\mu$ equivalent to the existence of a $G$-equivariant thinning from $\lambda$ to $\mu$ ? As seen above, this property holds for $S^{1}$ with rotations (Theorem 11) and for the ball with isometries, but not for $S^{1}$ with isometries (Remark [6).

6.3. Splitting. We say that a deterministic thinning $f$ on $[0,1]$ from $\lambda$ to $\mu$ is a $(\boldsymbol{\lambda}, \boldsymbol{\mu})$-splitting if $f(\Pi)$ and $\Pi-f(\Pi)$ are both Poisson point processes on $[0,1]$, with respective intensities $\mu$ and $\lambda-\mu$. The existence of a $(\lambda, \mu)$-splitting implies but is not equivalent to the existence of both a thinning from $\lambda$ to $\mu$ and a thinning from $\lambda$ to $\lambda-\mu$.

Question 4. Give necessary and sufficient conditions on $(\lambda, \mu)$ for the existence of a $(\lambda, \mu)$-splitting.

\section{ACKNOWLEDGMENT}

We thank Michael Brand for valuable comments. 


\section{REFERENCES}

[1] K. Ball. Poisson thinning by monotone factors. Electron. Comm. Probab., 10:60-69, 2005. MR 2133893 (2005m:60106)

[2] M. Brand. Using Your Head Is Permitted. http://www.brand.site.co.il/riddles, March 2009.

[3] S. Evans. A zero-one law for linear transformations of Lévy noise. In Proceedings of the Conference on Algebraic Methods in Statistics and Probability, Contemporary Mathematics Series, 2010. To appear.

[4] O. Gurel-Gurevich and R. Peled. Poisson thickening. Preprint, arXiv:0911.5377.

[5] A. E. Holroyd and Y. Peres. Extra heads and invariant allocations. Ann. Probab., 33(1):31-52, 2005. MR2118858 (2005k:60153)

[6] J. F. C. Kingman. Poisson Processes, volume 3 of Oxford Studies in Probability. The Clarendon Press, Oxford University Press, New York, 1993. Oxford Science Publications. MR.1207584 (94a:60052)

[7] R. Lyons, A. Holroyd, and T. Soo. Poisson splitting by factors. Preprint, arXiv:0908.3409.

[8] R.-D. Reiss. A Course on Point Processes. Springer Series in Statistics. Springer-Verlag, New York, 1993. MR1199815 (94b:60058)

[9] F. Spitzer. A combinatorial lemma and its application to probability theory. Trans. Amer. Math. Soc., 82:323-339, 1956. MR0079851(18:156e)

[10] S. M. Srivastava. A Course on Borel Sets, volume 180 of Graduate Texts in Mathematics. Springer-Verlag, New York, 1998. MR1619545 (99d:04002)

[11] V. Strassen. The existence of probability measures with given marginals. Ann. Math. Statist, 36:423-439, 1965. MR0177430 (31:1693)

[12] H. Thorisson. Coupling, Stationarity, and Regeneration. Probability and its Applications (New York). Springer-Verlag, New York, 2000. MR1741181 (2001b:60003)

[13] P. Winkler. Mathematical Puzzles: A Connoisseur's Collection. A K Peters Ltd., Natick, MA, 2004. MR2034896 (2006c:00002)

Department of Mathematics, University of British Columbia, 121-1984 Mathematics Road, Vancouver, BC V6T 1Z2, Canada

E-mail address: angel@math.ubc.ca

Microsoft Research, 1 Microsoft Way, Redmond, Washington 98052

E-mail address: holroyd@math.ubc.ca

Department of Mathematics, University of British Columbia, 121-1984 Mathematics

Road, Vancouver, BC V6T 1Z2, CANada

E-mail address: tsoo@math.ubc.ca 